\title{
Integrable Hierarchy of the Quantum Benjamin-Ono Equation ${ }^{\star}$
}

Maxim NAZAROV and Evgeny SKLYANIN

Department of Mathematics, University of York, York YO10 5DD, United Kingdom

Received September 26, 2013, in final form December 03, 2013; Published online December 07, 2013 http://dx.doi.org/10.3842/SIGMA.2013.078

\begin{abstract}
A hierarchy of pairwise commuting Hamiltonians for the quantum periodic Benjamin-Ono equation is constructed by using the Lax matrix. The eigenvectors of these Hamiltonians are Jack symmetric functions of infinitely many variables $x_{1}, x_{2}, \ldots$. This construction provides explicit expressions for the Hamiltonians in terms of the power sum symmetric functions $p_{n}=x_{1}^{n}+x_{2}^{n}+\cdots$ and is based on our recent results from [Comm. Math. Phys. 324 (2013), 831-849].
\end{abstract}

Key words: Jack symmetric functions; quantum Benjamin-Ono equation; collective variables

2010 Mathematics Subject Classification: 33D52; 05E05; 37K10; 81Q80

\section{Introduction}

Take the ring $\mathbb{C}\left[p_{1}, p_{2}, \ldots\right]$ of polynomials in countably many variables $p_{1}, p_{2}, \ldots$ and let $\alpha \in \mathbb{C}$ be a parameter. For $n=1,2, \ldots$ denote by $p_{n}^{*}$ the operator $\alpha n \partial / \partial p_{n}$ of renormalized differentiation relative to the variable $p_{n}$. The following differential operator on $\mathbb{C}\left[p_{1}, p_{2}, \ldots\right]$ arises in various problems of algebra and mathematical physics and has recently enjoyed considerable attention:

$$
\sum_{m, n \geqslant 1}\left(p_{m} p_{n} p_{m+n}^{*}+p_{m+n} p_{m}^{*} p_{n}^{*}\right)+(\alpha-1) \sum_{n \geqslant 1} n p_{n} p_{n}^{*}
$$

see $[2,4,9,11,13]$ for instance. In the classical limit $\alpha \rightarrow 0$, the parameter $\alpha$ playing the role of the Planck constant, the operator (1.1) becomes the Hamiltonian of the periodic Benjamin-Ono equation, a well-studied integrable Hamiltonian system [1, 3, 5, 6, 12].

The ring $\mathbb{C}\left[p_{1}, p_{2}, \ldots\right]$ can be identified with the ring of symmetric functions in the variables $x_{1}, x_{2}, \ldots$ by setting $p_{n}=x_{1}^{n}+x_{2}^{n}+\cdots$ for every $n$. It is known that then the operator (1.1) is diagonalised in the basis of the Jack symmetric functions corresponding to the parameter $\alpha$. It is also known [14] that (1.1) can be included into an infinite family of commuting operators, all diagonal in the basis of Jack symmetric functions. In [9] we provided an explicit expression for a generating function $A(u)$ of those commuting operators, see Theorem 1 in Section 4 of the present article. The function $A(u)$ has been defined as the projective limit of the renormalized Sekiguchi-Debiard determinant for the $N$-particle Calogero-Sutherland model as $N \rightarrow \infty$.

The purpose of the present article is to construct a generating function $I(u)$ of another family $I^{(1)}, I^{(2)}, \ldots$ of commuting operators diagonal in the basis of the Jack symmetric functions. Our construction is drastically different from that of [9] and has been inspired by the Lax formulation of the classical Benjamin-Ono equation. Namely, put

$$
I(u)=I^{(1)} u^{-1}+I^{(2)} u^{-2}+\cdots,
$$

${ }^{\star}$ This paper is a contribution to the Special Issue in honor of Anatol Kirillov and Tetsuji Miwa. The full collection is available at http://www.emis.de/journals/SIGMA/InfiniteAnalysis2013.html 
where by definition

$$
1-u^{-1} I(u)=A(u) / A(u+1)
$$

Our main result is Theorem 2 in Section 4 giving an explicit formula for $I(u)$. In particular $I^{(1)}$ is the weight counting operator $p_{1} p_{1}^{*}+p_{2} p_{2}^{*}+\cdots$ while $I^{(2)}$ coincides with the operator (1.1).

An advantage of this new generating function $I(u)$ is that it is written explicitly in terms of the collective variables $p_{1}, p_{2}, \ldots$ whereas $A(u)$ is written in terms of the monomial symmetric functions, which are expressed in these variables in a rather complicated combinatorial way.

Here is the plan of this article. In Section 2, following a brief discussion of the known results on the classical Benjamin-Ono equation, we explain our motivation and the origin of the formula (6.3). In Sections 3 and 4 we recall the basic facts about symmetric functions that we need for our proofs. In Section 5 we outline the results of [9] and describe the generating function $A(u)$. In Section 6 we state our main result, Theorem 2. In Section 7 we introduce our main tool, the Baker-Akhiezer vector (7.1). In Section 8 we complete the proof. Its main idea is to write (7.1) in terms of the monomial symmetric functions similarly to $A(u)$, see Theorem 3 in Section 7.

In this article we generally keep to the notation of the book [8] for symmetric functions. When using results from [8] we will simply indicate their numbers within the book. For example, the statement (6.19) from Chapter I of the book will be referred to as [I.6.9] assuming it is from [8].

\section{Classical periodic Benjamin-Ono equation}

Let $s \in \mathbb{R}$ be a real variable. The classical Benjamin-Ono equation $[1,3,5,6,12]$ is formulated in terms of the real-valued field $\theta(s)$ on the line $\mathbb{R}$ with the Poisson bracket

$$
\{\theta(s), \theta(t)\}=2 \pi \delta^{\prime}(s-t)
$$

Imposing the periodicity condition $s \equiv s+2 \pi$ and setting $z=\mathrm{e}^{-\mathrm{i} s}$ one can identify $\mathbb{R} / 2 \pi \mathbb{Z}$ with the unit circle $S^{1}=\{z \in \mathbb{C}:|z|=1\}$. Respectively, set $\varphi(z)=\theta(s)$. Let $p_{0}$ and $p_{n}, \bar{p}_{n}$ be the Fourier coefficients of the function $\varphi(z)$ :

$$
\varphi(z)=p_{0}+\sum_{n \geqslant 1}\left(p_{n} z^{-n}+\bar{p}_{n} z^{n}\right) .
$$

In terms of these coefficients the Poisson bracket (2.1) reads as follows:

$$
\begin{aligned}
& \left\{p_{m}, p_{n}\right\}=\left\{\bar{p}_{m}, \bar{p}_{n}\right\}=0 \quad \text { and } \quad\left\{\bar{p}_{m}, p_{n}\right\}=\mathrm{i} n \delta_{m n} \quad \text { for } \quad m, n \geqslant 1 \\
& \left\{p_{0}, p_{n}\right\}=\left\{p_{0}, \bar{p}_{n}\right\}=0 \quad \text { for } \quad n \geqslant 0 .
\end{aligned}
$$

Since $p_{0}$ belongs to the center of the Poisson bracket, and since the Hamiltonians we are interested in do not depend on $p_{0}$, one can safely set $p_{0}=0$. We will do so henceforth.

Introduce the projections $\varphi \mapsto \varphi_{ \pm}$where

$$
\varphi_{+}(z)=\sum_{n \geqslant 1} \bar{p}_{n} z^{n} \quad \text { and } \quad \varphi_{-}(z)=\sum_{n \geqslant 1} p_{n} z^{-n}
$$

Note that the constant term $p_{0}$ is now excluded. Consider the periodic Hilbert transform $\mathcal{H}$,

$$
(\mathcal{H} \varphi)(z)=-\mathrm{i} \varphi_{+}(z)+\mathrm{i} \varphi_{-}(z)=\text { p.v. } \int_{S^{1}} \frac{\mathrm{d} t}{2 \pi} \cot \frac{s-t}{2} \theta(t) .
$$


The periodic Benjamin-Ono equation is then characterized by the Hamiltonian

$$
\mathcal{I}=\int_{S^{1}} \frac{\mathrm{d} s}{2 \pi}\left(\frac{1}{3} \theta^{3}(s)+\frac{1}{2} \theta^{\prime}(s)(\mathcal{H} \theta)(s)\right)=\sum_{m, n \geqslant 1}\left(p_{m} p_{n} \bar{p}_{m+n}+p_{m+n} \bar{p}_{m} \bar{p}_{n}\right)-\sum_{n \geqslant 1} n p_{n} \bar{p}_{n}
$$

which is simply the classical limit (as $\alpha \rightarrow 0$ ) of the operator (1.1). The correspondence rule between the quantum commutator and classical Poisson bracket we use here is $\alpha^{-1}[,] \rightarrow \mathrm{i}\{$,$\} .$

In the classical case, the equations of motion determined by the Hamiltonian $\mathcal{I}$ read

$$
\partial \varphi(z) / \partial t=\{\varphi(z), \mathcal{I}\}=\mathrm{i}(z \partial / \partial z)^{2}\left(\varphi_{+}-\varphi_{-}\right)(z)-\mathrm{i}(z \partial / \partial z) \varphi^{2}(z)
$$

or equivalently,

$$
\begin{aligned}
& \partial p_{n} / \partial t=-\mathrm{i} n^{2} p_{n}+2 \mathrm{i} n \sum_{m \geqslant 1} p_{n+m} \bar{p}_{m}+\mathrm{i} n \sum_{1 \leqslant m<n} p_{m} p_{n-m}, \\
& \partial \bar{p}_{n} / \partial t=\mathrm{i} n^{2} \bar{p}_{n}-2 \mathrm{i} n \sum_{m \geqslant 1} \bar{p}_{n+m} p_{m}-\mathrm{i} n \sum_{1 \leqslant m<n} \bar{p}_{m} \bar{p}_{n-m} .
\end{aligned}
$$

The Benjamin-Ono equation is known to be a completely integrable system having a countable set of higher commuting Hamiltonians $[1,5,6]$. The key to the integrability of the equation (2.2) is given by its Lax representation

$$
\partial \mathcal{L} / \partial t=[\mathcal{M}, \mathcal{L}]
$$

where the Lax operators $\mathcal{L}$ and $\mathcal{M}$ are the integro-differential operators acting on the functions

$$
f(z)=f_{0}+f_{1} z+f_{2} z^{2}+\cdots
$$

and are defined by

$$
\begin{aligned}
& \mathcal{L}: f \mapsto-z \partial f / \partial z+(\varphi f)_{+}, \\
& \mathcal{M}: f \mapsto \mathrm{i}(z \partial / \partial z)^{2} f-2 \mathrm{i} \varphi_{+} z \partial f / \partial z-2 \mathrm{i}\left(z \partial\left(\varphi_{-} f\right) / \partial z\right)_{+} .
\end{aligned}
$$

The functions $f(z)$ satisfying the condition $f(0)=0$ consitute an invariant subspace for the operators $\mathcal{L}$ and $\mathcal{M}$ that is more convenient for us to use. Identifying $f(z)$ with the column vector

$$
\left[\begin{array}{c}
f_{1} \\
f_{2} \\
\vdots
\end{array}\right]
$$

one can represent the operators $\mathcal{L}$ and $\mathcal{M}$ by the infinite matrices as follows:

$$
\mathcal{L}=\left[\begin{array}{ccccc}
-1 & p_{1} & p_{2} & p_{3} & \ldots \\
\bar{p}_{1} & -2 & p_{1} & p_{2} & \ddots \\
\bar{p}_{2} & \bar{p}_{1} & -3 & p_{1} & \ddots \\
\vdots & \ddots & \ddots & \ddots & \ddots
\end{array}\right], \quad \mathcal{M}=\mathrm{i}\left[\begin{array}{ccccc}
1 & -2 p_{1} & -2 p_{2} & -2 p_{3} & \cdots \\
-2 \bar{p}_{1} & 4 & -4 p_{1} & -4 p_{2} & \ddots \\
-2 \bar{p}_{2} & -4 \bar{p}_{1} & 9 & -6 p_{1} & \ddots \\
\vdots & \ddots & \ddots & \ddots & \ddots
\end{array}\right]
$$

It is easy to verify that the equation (2.5) for these matrices is equivalent to (2.3) and (2.4).

The most common way to produce the commuting integrals of motion for an integrable system is to define them as coefficients of expansion in $u$ of the spectral determinant $\operatorname{det}(u-\mathcal{L})$, or equivalently, of its logarithmic derivative $\operatorname{tr}(u-\mathcal{L})^{-1}$. But our operator $\mathcal{L}$ is a pertubation of the differential operator $-z \partial / \partial z$ with a Toeplitz matrix, and its spectrum is unbounded. The 
above determinant and trace both diverge if understood literally. Although it might be possible to regularize them, we shall take another approach by using a peculiarity of the Benjamin-Ono equation, namely the fact that it has a rational spectral curve.

The integrable systems with a rational spectral curve, for example the $N$-particle open Toda chain and the Calogero-Moser model, possess peculiar properties that are missing for non-zero genus spectral curves. In particular, instead of $\operatorname{tr}(u-\mathcal{L})^{-1}$ one can use, as a generating function of integrals of motion, the matrix element $r(u-\mathcal{L})^{-1} c$ for a special pair $r$ and $c$ of row and column vectors. See $[7,16,18]$ for the open Toda chain and $[18,20]$ for the Calogero-Moser model. In our case, the appropriate vectors are $r=p=\left[\begin{array}{lll}p_{1} & p_{2} & \ldots\end{array}\right]$ and its conjugate

$$
c=\bar{p}=\left[\begin{array}{c}
\bar{p}_{1} \\
\bar{p}_{2} \\
\vdots
\end{array}\right] .
$$

It is easy to show that the coefficients of the generating function

$$
\mathcal{I}(u)=p(u-\mathcal{L})^{-1} \bar{p}
$$

commute with the Hamiltonian $\mathcal{I}$ relative to the Poisson bracket. Here the identity $\partial \mathcal{I}(u) / \partial t=0$ follows immediately from (2.5) and from the equalities

$$
\{\bar{p}, \mathcal{I}\}=\mathcal{M} \bar{p} \quad \text { and } \quad\{p, \mathcal{I}\}=-p \mathcal{M}
$$

which can be easily verified. We do not discuss now the pairwise commutativity of all coefficients of $\mathcal{I}(u)$ since we are going to prove it in the quantum case, thus obtaining the commutativity in the classical case as a byproduct.

A natural question is whether there exists a quantum analog of the formula (2.6). A strong indication that this might be the case is provided by the example of the $N$-particle quantum Calogero-Moser system [19] where the quantum Lax matrix and the vectors $r, c$ coincide with the classical ones. We answer this question affirmatively. Namely, the quantum deformations of the Lax operator $\mathcal{L}$ and of the generating function $\mathcal{I}(u)$ are given, respectively, by (6.1) and (6.3).

\section{Monomial symmetric functions and powers sums}

Fix any field $\mathbb{F}$. Denote by $\Lambda$ the $\mathbb{F}$-algebra of symmetric functions in infinitely many variables $x_{1}, x_{2}, \ldots$. Following [8] we will introduce some standard bases of $\Lambda$.

Let $\lambda=\left(\lambda_{1}, \lambda_{2}, \ldots\right)$ be any partition of $0,1,2, \ldots$. Here we assume that $\lambda_{1} \geqslant \lambda_{2} \geqslant \cdots$. The number of non-zero parts is called the length of $\lambda$ and denoted by $\ell(\lambda)$. We will also denote $|\lambda|=\lambda_{1}+\lambda_{2}+\cdots$ as usual. Let $m_{\lambda} \in \Lambda$ be the monomial symmetric function corresponding to the partition $\lambda$. By definition,

$$
m_{\lambda}=\sum_{i_{1}<\cdots<i_{k}} \sum_{\sigma \in \mathfrak{S}_{k}} d_{\lambda}^{-1} x_{i_{\sigma(1)}}^{\lambda_{1}} \cdots x_{i_{\sigma(k)}}^{\lambda_{k}},
$$

where we write $k$ instead of $\ell(\lambda)$. Here $\mathfrak{S}_{k}$ is the symmetric group permuting $1, \ldots, k$ while

$$
d_{\lambda}=k_{1} ! k_{2} ! \cdots
$$

if $k_{1}, k_{2}, \ldots$ are the respective multiplicites of the parts $1,2, \ldots$ of $\lambda$. If $\ell(\lambda)=0$ then $m_{\lambda}=1$. The monomial symmetric functions corresponding to partitions of $0,1,2, \ldots$ form a basis of $\Lambda$.

For $n=1,2, \ldots$ let $p_{n} \in \Lambda$ be the power sum symmetric function of degree $n$. By definition,

$$
p_{n}=x_{1}^{n}+x_{2}^{n}+\cdots \text {. }
$$


More generally, for any partition $\lambda$ put $p_{\lambda}=p_{\lambda_{1}} \cdots p_{\lambda_{k}}$ where $k=\ell(\lambda)$ as in (3.1). The elements $p_{\lambda}$ form another basis of $\Lambda$. In other words, the elements $p_{1}, p_{2}, \ldots$ are free generators of the commutative algebra $\Lambda$ over $\mathbb{F}$. We will also use the formal power series in the variable $t$

$$
P(t)=p_{1}+p_{2} t+p_{3} t^{2}+\cdots .
$$

Define a bilinear form $\langle$,$\rangle on the vector space \Lambda$ by setting for any two partitions $\lambda$ and $\mu$

$$
\left\langle p_{\lambda}, p_{\mu}\right\rangle=z_{\lambda} \delta_{\lambda \mu}, \quad \text { where } \quad z_{\lambda}=1^{k_{1}} k_{1} ! 2^{k_{2}} k_{2} ! \cdots
$$

in the notation (3.2). This form is obviously symmetric and non-degenerate. We will indicate by the superscript ${ }^{\perp}$ the operator conjugation relative to this bilinear form. In particular, by (3.4) for the operator conjugate to the multiplication in $\Lambda$ by $p_{n}$ with $n \geqslant 1$ we have $p_{n}^{\perp}=n \partial / \partial p_{n}$.

The basis of $p_{\lambda}$ is related to the basis of monomial symmetric functions as follows. For any two partitions $\lambda$ and $\mu$ let $r_{\lambda \mu}$ be the number of maps $\theta:\{1, \ldots, \ell(\mu)\} \rightarrow\{1,2, \ldots\}$ such that

$$
\sum_{\theta(j)=i} \mu_{j}=\lambda_{i} \quad \text { for each } \quad i=1,2, \ldots
$$

For any such $\theta$ the partition $\mu$ in (3.5) is called a refinement of $\lambda$. Note that if $r_{\lambda \mu} \neq 0$ then $|\lambda|=|\mu|$. Moreover, then by [I.6.10] we have $\mu \leqslant \lambda$ in the natural partial ordering of partitions:

$$
\mu_{1} \leqslant \lambda_{1}, \quad \mu_{1}+\mu_{2} \leqslant \lambda_{1}+\lambda_{2}, \quad \ldots
$$

By [I.6.9] we have the relation

$$
p_{\mu}=\sum_{\mu} r_{\lambda \mu} m_{\lambda}
$$

\section{Elementary and complete symmetric functions}

For $n=1,2, \ldots$ let $e_{n} \in \Lambda$ be the elementary symmetric function of degree $n$. By definition,

$$
e_{n}=\sum_{i_{1}<\cdots<i_{k}} x_{i_{1}} \cdots x_{i_{k}}
$$

Note that by the definition (3.1) we have $e_{n}=m_{\lambda}$ where $\lambda=\left(1^{n}\right)$. Further, let $h_{n} \in \Lambda$ be the complete symmetric function of degree $n$. By definition,

$$
h_{n}=\sum_{|\lambda|=n} m_{\lambda}
$$

We will also use the formal power series in the variable $t$

$$
E(t)=1+e_{1} t+e_{2} t^{2}+\cdots \quad \text { and } \quad H(t)=1+h_{1} t+h_{2} t^{2}+\cdots .
$$

In particular, by [I.2.6] we have the relation

$$
E(-t) H(t)=1 \text {. }
$$

Further, by [I.2.10] we have

$$
P(t)=E^{\prime}(-t) / E(-t) \text {. }
$$


For any partition $\lambda$ denote $e_{\lambda}=e_{\lambda_{1}} e_{\lambda_{2}} \cdots$ and $h_{\lambda}=h_{\lambda_{1}} h_{\lambda_{2}} \cdots$ where $e_{0}=h_{0}=1$. The elements $e_{\lambda}$ form a basis of $\Lambda$, and so do the elements $h_{\lambda}$. It follows from (4.1) that for any $n \geqslant 1$

$$
(-1)^{n} e_{n}=\sum_{|\lambda|=n}(-1)^{\ell(\lambda)} h_{\lambda} \ell(\lambda) ! / d_{\lambda}
$$

Furthermore, according to [I.4.5] the bases of $m_{\lambda}$ and $h_{\lambda}$ are dual to each other relative to the bilinear form (3.4) on $\Lambda$ :

$$
\left\langle m_{\lambda}, h_{\mu}\right\rangle=\delta_{\lambda \mu} .
$$

It follows from this duality that for any partitions $\lambda$ and $\nu$ we have

$$
h_{\nu}^{\perp}\left(m_{\lambda}\right)= \begin{cases}m_{\mu} & \text { if } \lambda=\mu \sqcup \nu, \\ 0 & \text { otherwise }\end{cases}
$$

where $\mu \sqcup \nu$ is the partition obtained by collecting the parts of $\mu$ and $\nu$ together. For the operator $p_{n}^{\perp}$ with $n \geqslant 1$ acting on the elements of $\Lambda$ expressed as polynomials in $h_{1}, h_{2}, \ldots$ by [Ex. I.5.3]

$$
p_{n}^{\perp}=\sum_{k \geqslant 0} h_{k} \partial / \partial h_{n+k} .
$$

\section{Jack symmetric functions}

Now let $\mathbb{F}$ be the field $\mathbb{Q}(\alpha)$ where $\alpha$ is another variable. Generalizing (3.4) define a bilinear form $\langle,\rangle_{\alpha}$ on $\Lambda$ by setting for any partitions $\lambda$ and $\mu$

$$
\left\langle p_{\lambda}, p_{\mu}\right\rangle_{\alpha}=\alpha^{\ell(\lambda)} z_{\lambda} \delta_{\lambda \mu} .
$$

This form is symmetric and non-degenerate. In particular, if $\alpha=1$ then this is the form $\langle$,$\rangle .$ We will indicate by the superscript * the operator conjugation relative to the form $\langle,\rangle_{\alpha}$. Using the definition (5.1) for the operator conjugate to the multiplication in $\Lambda$ by $p_{n}$ with $n \geqslant 1$ we get

$$
p_{n}^{*}=\alpha n \partial / \partial p_{n}
$$

By [Ex. VI.4.2] there exists a unique family of elements $J_{\lambda} \in \Lambda$ such that

$$
\left\langle J_{\lambda}, J_{\mu}\right\rangle_{\alpha}=0 \quad \text { for } \quad \lambda \neq \mu
$$

and such that the $J_{\lambda}$ equals $m_{\lambda}$ plus a linear combination of the elements $m_{\mu}$ with $\mu<\lambda$ in the natural partial ordering. The elements $J_{\lambda} \in \Lambda$ are called the Jack symmetric functions. In the case $\alpha=1$ the $J_{\lambda}$ coincides with the Schur symmetric function $s_{\lambda}$.

The Jack symmetric functions $J_{\lambda}$ form a basis of the vector space $\Lambda$ over the field $\mathbb{Q}(\alpha)$. Let us now define the operators $A^{(1)}, A^{(2)}, \ldots$ acting on $\Lambda$ as follows. Put

$$
A(u)=1+A^{(1)} /(u)_{1}+A^{(2)} /(u)_{2}+\cdots,
$$

where $u$ is another variable and $(u)_{k}=u(u+1) \cdots(u+k-1)$ is the Pochhammer symbol. Then

$$
A(u) J_{\lambda}=\prod_{i \geqslant 1} \frac{u+i-1-\alpha \lambda_{i}}{u+i-1} \cdot J_{\lambda}
$$

by definition. Note that in the infinite product displayed above the only factors different from 1 are those corresponding to $i=1, \ldots, \ell(\lambda)$. In our recent publication [9] we proved the following theorem. Another proof can be obtained by using the results of [15], see also [10]. 
Theorem 1. In the notation (3.2) for each $k=1,2, \ldots$ we have

$$
A^{(k)}=(-1)^{k} \sum_{\ell(\lambda)=k} d_{\lambda} m_{\lambda} m_{\lambda}^{*}
$$

where the sum is taken over all partitions $\lambda$ of the fixed length $k$.

The Jack symmetric functions are eigenvectors of the operators $A^{(1)}, A^{(2)}, \ldots$ by definition. In particular, these operators are self-conjugate relative to the form $\langle,\rangle_{\alpha}$ by the orthogonality condition (5.3). This self-conjugacy is also transparent from the explicit formula (5.6).

By inverting the relation (3.6) any monomial symmetric function $m_{\lambda}$ can be expressed as a linear combination of the functions $p_{\mu}$ where $\lambda, \mu$ are partitions of the same number and $\lambda \leqslant \mu$. By substituting into (5.6) and using (5.2), one can write each operator $A^{(k)}$ in terms of $p_{n}$ and $\partial / \partial p_{n}$ where $n=1,2, \ldots$. However, the result of this procedure for arbitrary $k$ does not seem to be sufficiently explicit. In the next section we will overcome this difficulty by considering another collection of operators on $\Lambda$ such that the Jack symmetric functions are their eigenvectors.

\section{Lax matrix}

Let us define the operators $I^{(1)}, I^{(2)}, \ldots$ acting on the vector space $\Lambda$ over $\mathbb{Q}(\alpha)$ by the equations (1.2) and (1.3). In particular, then we have the relations

$$
I^{(1)}=-A^{(1)} \quad \text { and } \quad I^{(2)}=A^{(1)}\left(A^{(1)}+1\right)-2 A^{(2)} .
$$

Note that then due to the definition (5.5) of $A(u)$ we also have the equality

$$
I(u) J_{\lambda}=u J_{\lambda}-(u+\ell(\lambda)) \prod_{1 \leqslant i \leqslant \ell(\lambda)} \frac{u+i-1-\alpha \lambda_{i}}{u+i-\alpha \lambda_{i}} \cdot J_{\lambda} .
$$

Let us now introduce the infinite matrix

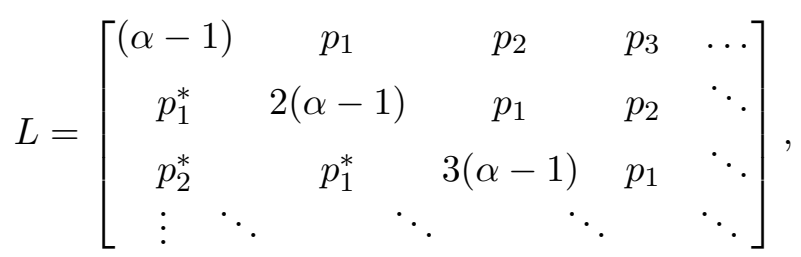

where the rows and columns can be labelled by the indices $i, j=1,2, \ldots$. We shall call it the Lax matrix. It is convenient to set $p_{-n}=p_{n}^{*}$ for $n \geqslant 1$. We also keep assuming that $p_{0}=0$. Then $L=\left[L_{i j}\right]_{i, j=1}^{\infty}$ where

$$
L_{i j}=j(\alpha-1) \delta_{i j}+p_{j-i} .
$$

Note that the matrix $L$ is self-conjugate relative to the form $\langle,\rangle_{\alpha}$. Also introduce the infinite row vector $p=\left[\begin{array}{lll}p_{1} & p_{2} & \ldots\end{array}\right]$ and its conjugate column vector

$$
p^{*}=\left[\begin{array}{c}
p_{1}^{*} \\
p_{2}^{*} \\
\vdots
\end{array}\right]
$$

The main result of the present article is the next theorem which provides an explicit expression for every operator $I^{(k)}$ in terms of $p_{n}$ and $\partial / \partial p_{n}$ where $n=1,2, \ldots$ 
Theorem 2. We have the equality

$$
I(u)=p(u-L)^{-1} p^{*},
$$

where the inverse to the infinite matrix $u-L$ is regarded as formal power series in $u^{-1}$ :

$$
(u-L)^{-1}=u^{-1}+L u^{-2}+L^{2} u^{-3}+\cdots .
$$

Note that each coefficient of the series in $u^{-1}$ at the right hand side of the equality (6.3) is an infinite sum of operators acting on $\Lambda$. However, only finite number of the summands do not vanish on any subspace in $\Lambda$ of a fixed degree in $x_{1}, x_{2}, \ldots$. This can be easily seen by restating Theorem 2 as the equality for every index $k \geqslant 1$

$$
I^{(k)}=\sum_{i_{1}, \ldots, i_{k}=1}^{\infty} p_{i_{1}} L_{i_{1} i_{2}} \cdots L_{i_{k-1} i_{k}} p_{i_{k}}^{*}
$$

and by performing summation in (6.4) consecutively over the indices $i_{k}, i_{k-1}, \ldots, i_{1}$. In the next section we explain our method of proving Theorem 2. The proof will be completed after that.

\section{Baker-Akhiezer vector}

Consider the infinite column vector

$$
\Psi(u)=\left[\begin{array}{c}
\Psi_{1}(u) \\
\Psi_{2}(u) \\
\vdots
\end{array}\right]=(u-L)^{-1} p^{*} A(u+1) .
$$

Every entry $\Psi_{n}(u)$ of this vector is a formal power series in $u^{-1}$ with coefficients acting on $\Lambda$; see the remark in the end of previous section. We shall call it the Baker-Akhiezer vector for the Lax matrix (6.1), since in the classical limit this is an analog of the Baker-Akhiezer function for a rational spectral curve [16]. Our proof of Theorem 2 is based on the explicit formula for $\Psi_{n}(u)$ given by the next theorem. This theorem is another principal result of the present article. Let

$$
\Psi_{n}(u)=\Psi_{n}^{(1)} /(u+1)_{1}+\Psi_{n}^{(2)} /(u+1)_{2}+\cdots,
$$

where the coefficients $\Psi_{n}^{(1)}, \Psi_{n}^{(2)}, \ldots$ are operators acting on the vector space $\Lambda$ over $\mathbb{Q}(\alpha)$.

Theorem 3. For any indices $k, n \geqslant 1$ we have the equality

$$
\Psi_{n}^{(k)}=(-1)^{n+k} \sum_{\ell(\lambda)=k} d_{\lambda} e_{n}^{\perp}\left(m_{\lambda}\right) m_{\lambda}^{*} .
$$

Note that by using the relation (4.3) and then (4.5) here we can write

$$
(-1)^{n} e_{n}^{\perp}\left(m_{\lambda}\right)=\sum_{\substack{\lambda=\mu \sqcup \nu \\|\nu|=n}}(-1)^{\ell(\nu)} m_{\mu} \ell(\nu) ! / d_{\nu},
$$

where $\nu$ ranges over all partions of $n$ such that by taking the parts of $\nu$ together with those of another partition $\mu$ we get $\lambda$. The partition $\mu$ is then determined by $\lambda$ and $\nu$ uniquely.

Theorem 3 shows that the series $\Psi_{n}(u)$ can be obtained from the series $A(u+1)$ by applying the operator $(-1)^{n} e_{n}^{\perp}$ to the factor $m_{\lambda}$ in the coefficient (5.6). Thus $\Psi_{n}(u)$ can be expressed in terms of $A(u+1)$ without using Theorem 1. However we will prove Theorem 3 as stated here. 
Theorem 2 will then follow rather easily. Indeed, due to the definition (7.1) of $\Psi(u)$ to obtain the stated equality (6.3) it suffices to prove that

$$
p \Psi(u)=I(u) A(u+1) .
$$

By using the definition (1.3) of $I(u)$ the last displayed equality can be rewritten as

$$
p \Psi(u)=u A(u+1)-u A(u) .
$$

Here on the left hand side we have a product of a row vector by a column vector:

$$
p \Psi(u)=\sum_{n \geqslant 1} p_{n} \Psi_{n}(u)
$$

By using Theorems 1 and 3 along with the obvious relation for any $k \geqslant 1$

$$
u /(u+1)_{k}-u /(u)_{k}=-k /(u+1)_{k},
$$

the equality (7.4) follows from the proposition which is stated and proved below.

Proposition 1. For any partition $\lambda$ of the length $k \geqslant 1$ we have

$$
\sum_{n \geqslant 1}(-1)^{n} p_{n} e_{n}^{\perp}\left(m_{\lambda}\right)=-k m_{\lambda} .
$$

Proof. Using the bilinear form $\langle$,$\rangle take the operator on \Lambda$ conjugate to the operator which is applied to $m_{\lambda}$ at the left hand side of the equality (7.5). The conjugate operator equals

$$
\begin{aligned}
\sum_{n \geqslant 1}(-1)^{n} e_{n} p_{n}^{\perp} & =\sum_{n \geqslant 1} \sum_{k \geqslant 0}(-1)^{n} e_{n} h_{k} \partial / \partial h_{n+k} \\
& =\sum_{n \geqslant 1} \sum_{0 \leqslant k<n}(-1)^{n-k} e_{n-k} h_{k} \partial / \partial h_{n}=-\sum_{n \geqslant 1} h_{n} \partial / \partial h_{n},
\end{aligned}
$$

where we used the relation (4.6) and then (4.1). Therefore by applying the conjugate operator to the vector $h_{\lambda}$ with $\ell(\lambda)=k$ we get $-k h_{\lambda}$. Now (7.5) follows from the duality relation (4.4).

\section{Proof of Theorem 3}

Denote by $\Phi_{n}^{(k)}$ the right hand side of the relation (7.2). Then introduce the series

$$
\Phi_{n}(u)=\Phi_{n}^{(1)} /(u+1)_{1}+\Phi_{n}^{(2)} /(u+1)_{2}+\cdots
$$

and the column vector

$$
\Phi(u)=\left[\begin{array}{c}
\Phi_{1}(u) \\
\Phi_{2}(u) \\
\vdots
\end{array}\right] .
$$

We have to prove the relation $\Psi(u)=\Phi(u)$. Multiplying by $u-L$ in the definition (7.1) of $\Psi(u)$ the latter relation becomes

$$
p^{*} A(u+1)=(u-L) \Phi(u)
$$


By taking the entries of the column vectors here and using the definition (6.2) we have to prove that for every index $n \geqslant 1$

$$
p_{n}^{*} A(u+1)=(u+n-\alpha n) \Phi_{n}(u)-\sum_{i \geqslant 1} p_{i-n} \Phi_{i}(u) .
$$

By using for $n \geqslant 1$ the expansions (5.4) and (8.1) along with the obvious relation for $k \geqslant 1$

$$
u /(u+1)_{k}=1 /(u+1)_{k-1}-k /(u+1)_{k},
$$

the relation (8.2) is equivalent to the collection of relations for all $k \geqslant 1$

$$
p_{n}^{*} A^{(k)}=\Phi_{n}^{(k+1)}+(n-\alpha n-k) \Phi_{n}^{(k)}-\sum_{i \geqslant 1} p_{i-n} \Phi_{n}^{(k)}
$$

together with the relation $p_{n}^{*}=\Phi_{n}^{(1)}$. But the latter relation follows from the definition of $\Phi_{n}^{(1)}$ by using (7.3). Indeed, if $\lambda=\mu \sqcup \nu$ where $\ell(\lambda)=1$ and $|\nu|=n \geqslant 1$, then $\lambda=\nu=(n)$ while $\mu$ has no parts different from zero. We then observe that $p_{n}^{*}=m_{(n)}^{*}$. So we have to prove (8.3).

For any two symmetric functions $f, g \in \Lambda$ the operator $f g^{*}$ acting on the vector space $\Lambda$ can be represented by its symbol $f \otimes g \in \Lambda \otimes \Lambda$. We will extend this representation to infinite sums of operators of the form $f g^{*}$ by linearity. In particular, the symbol of the operator at the right hand side of (5.6) is the infinite linear combination of elements of the vector space $\Lambda \otimes \Lambda$

$$
\Delta^{(k)}=(-1)^{k} \sum_{\ell(\lambda)=k} d_{\lambda} m_{\lambda} \otimes m_{\lambda} .
$$

Extending our notation, set $\Phi_{0}^{(k)}=A^{(k)}$. By Theorem 1 this is indeed the right hand side of (7.2) at $n=0$. Thus for any $n \geqslant 0$ the symbol of the operator $\Phi_{n}^{(k)}$ equals $(-1)^{n}\left(e_{n}^{\perp} \otimes 1\right) \Delta^{(k)}$.

Let us now prove (8.3) for $n, k \geqslant 1$. Using our extended notation, (8.3) can be rewritten as

$$
\sum_{1 \leqslant i \leqslant n} p_{i}^{*} \Phi_{n-i}^{(k)}+\sum_{i \geqslant 1} p_{i} \Phi_{n+i}^{(k)}=\Phi_{n}^{(k+1)}+(n-\alpha n-k) \Phi_{n}^{(k)} .
$$

In the representation by symbols the latter operator relation becomes

$$
\begin{gathered}
\sum_{1 \leqslant i \leqslant n}(-1)^{n-i}\left(\alpha p_{i}^{\perp} e_{n-i}^{\perp} \otimes 1+e_{n-i}^{\perp} \otimes p_{i}\right) \Delta^{(k)}+\sum_{i \geqslant 1}(-1)^{n+i}\left(p_{i} e_{n+i}^{\perp} \otimes 1\right) \Delta^{(k)} \\
=(-1)^{n}\left(e_{n}^{\perp} \otimes 1\right) \Delta^{(k+1)}+(n-\alpha n-k)(-1)^{n}\left(e_{n}^{\perp} \otimes 1\right) \Delta^{(k)} .
\end{gathered}
$$

Here we take commutators with $p_{i}^{*}$ and then use the equality $p_{i}^{*}=\alpha p_{i}^{\perp}$ given by (5.2).

Observe that both sides of the relation (8.5) are polynomials in $\alpha$ of degree 1. By equating their coefficients at $\alpha$ we get the relation

$$
\sum_{1 \leqslant i \leqslant n}(-1)^{n-i}\left(p_{i}^{\perp} e_{n-i}^{\perp} \otimes 1\right) \Delta^{(k)}=n(-1)^{n+1}\left(e_{n}^{\perp} \otimes 1\right) \Delta^{(k)} .
$$

But this relation follows from the identity

$$
\sum_{1 \leqslant i \leqslant n}(-1)^{n-i} e_{n-i} p_{i}=n(-1)^{n+1} e_{n}
$$

which holds by (4.2). It now remains to verify the relation (8.5) only for $\alpha=0$, that is

$$
\begin{gathered}
\sum_{1 \leqslant i \leqslant n}(-1)^{n-i}\left(e_{n-i}^{\perp} \otimes p_{i}\right) \Delta^{(k)}+\sum_{i \geqslant 1}(-1)^{n+i}\left(p_{i} e_{n+i}^{\perp} \otimes 1\right) \Delta^{(k)} \\
=(-1)^{n}\left(e_{n}^{\perp} \otimes 1\right) \Delta^{(k+1)}+(n-k)(-1)^{n}\left(e_{n}^{\perp} \otimes 1\right) \Delta^{(k)} .
\end{gathered}
$$


It follows from the definitions (3.1) and (3.3) that for any integer $i \geqslant 1$ and any partition $\mu$

$$
p_{i} m_{\mu}=\sum_{\lambda} c_{\lambda \mu} m_{\lambda}
$$

where $\lambda$ ranges over all those partitions which can be obtained by increasing by $i$ anyone of the distinct parts of $\mu$. That includes increasing a zero part of $\mu$, in which case $\ell(\lambda)=\ell(\mu)+1$. Otherwise we have $\ell(\lambda)=\ell(\mu)$ of course. In any case, the coefficient $c_{\lambda \mu}$ in (8.7) is equal to the multiplicity in $\lambda$ of that part which has been obtained by increasing. Note that if the increased part of $\mu$ is zero, that is if $\lambda=\mu \sqcup i$, then the product $c_{\lambda \mu} d_{\mu}$ equals $d_{\lambda}$. But if the increased part of $\mu$ is not zero, then the ratio $c_{\lambda \mu} d_{\mu} / d_{\lambda}$ equals the multiplicity of the increased part of $\mu$. Let us denote the latter multiplicity by $c_{\mu \lambda}$, so that we have $c_{\lambda \mu} d_{\mu}=c_{\mu \lambda} d_{\lambda}$ when $\ell(\lambda)=\ell(\mu)$.

The expansion (8.7) also shows that either side of the relation (8.6) can be written as a sum of tensor products of the form $f \otimes m_{\lambda}$ where $f \in \Lambda$ and $\ell(\lambda)=k, k+1$; see the definition (8.4). By taking only the summands $f \otimes m_{\lambda}$ in (8.6) where $\ell(\lambda)=k+1$, we get the relation

$$
\sum_{1 \leqslant i \leqslant n}(-1)^{n-i+k} \sum_{\ell(\mu)=k} c_{\mu \sqcup i, \mu} d_{\mu} e_{n-i}^{\perp}\left(m_{\mu}\right) \otimes m_{\mu \sqcup i}=(-1)^{n+k+1} \sum_{\ell(\lambda)=k+1} d_{\lambda} e_{n}^{\perp}\left(m_{\lambda}\right) \otimes m_{\lambda} .
$$

By using (4.5) and the equality $c_{\mu \sqcup i, \mu} d_{\mu}=d_{\mu \sqcup i}$ the last displayed relation be rewritten as

$$
\sum_{1 \leqslant i \leqslant n}(-1)^{n-i+k} \sum_{\ell(\lambda)=k+1} d_{\lambda} e_{n-i}^{\perp} h_{i}^{\perp}\left(m_{\lambda}\right) \otimes m_{\lambda}=(-1)^{n+k+1} \sum_{\ell(\lambda)=k+1} d_{\lambda} e_{n}^{\perp}\left(m_{\lambda}\right) \otimes m_{\lambda} .
$$

But this relation immediately follows from an identity which holds by (4.1),

$$
\sum_{1 \leqslant i \leqslant n}(-1)^{n-i} h_{i} e_{n-i}=(-1)^{n+1} e_{n} .
$$

Now take the summands $f \otimes m_{\lambda}$ in (8.6) where $\ell(\lambda)=k$. In this way we get the relation

$$
\begin{gathered}
\sum_{1 \leqslant i \leqslant n}(-1)^{n-i+k} \sum_{\ell(\mu)=k} \sum_{\lambda} c_{\lambda \mu} d_{\mu} e_{n-i}^{\perp}\left(m_{\mu}\right) \otimes m_{\lambda} \\
\quad+\sum_{i \geqslant 1} \sum_{\ell(\lambda)=k}(-1)^{n+i+k} d_{\lambda} p_{i} e_{n+i}^{\perp}\left(m_{\lambda}\right) \otimes m_{\lambda} \\
=(n-k)(-1)^{n+k} \sum_{\ell(\lambda)=k} d_{\lambda} e_{n}^{\perp}\left(m_{\lambda}\right) \otimes m_{\lambda},
\end{gathered}
$$

where in the first of the three displayed lines the partition $\lambda$ is obtained by increasing by $i$ any of the distinct non-zero parts of $\mu$. Let us consider the sum in the second line of the display (8.8).

Proposition 2. For any $n \geqslant 1$ we have the operator identity

$$
\sum_{i \geqslant 1}(-1)^{n+i} p_{i} e_{n+i}^{\perp}=\sum_{0 \leqslant i \leqslant n} \sum_{j \geqslant 1}(-1)^{n-i+1}\left(\partial / \partial h_{j}\right)^{\perp} e_{n-i}^{\perp} h_{i+j}^{\perp} .
$$

Proof. Using the form $\langle$,$\rangle let us conjugate the operator at the left hand side of (8.9). We get$

$$
\begin{aligned}
\sum_{i \geqslant 1}(-1)^{n+i} e_{n+i} p_{i}^{\perp} & =\sum_{i \geqslant 1} \sum_{l \geqslant 0}(-1)^{n+i} e_{n+i} h_{l} \partial / \partial h_{i+l}=\sum_{j \geqslant 1} \sum_{0 \leqslant l<j}(-1)^{n+j-l} e_{n+j-l} h_{l} \partial / \partial h_{j} \\
& =\sum_{j \geqslant 1} \sum_{j \leqslant l \leqslant n+j}(-1)^{n+j-l+1} e_{n+j-l} h_{l} \partial / \partial h_{j} \\
& =\sum_{j \geqslant 1} \sum_{0 \leqslant i \leqslant n}(-1)^{n-i+1} e_{n-i} h_{i+j} \partial / \partial h_{j}
\end{aligned}
$$

as required. Here we use (4.6), then denote $j=i+l$, then use (4.1), and finally set $i=l-j$. 
Further, the right hand side of the identity (8.9) can be rewritten as

$$
\sum_{0 \leqslant i \leqslant n} \sum_{j \geqslant 1}(-1)^{n-i+1} e_{n-i}^{\perp}\left(\partial / \partial h_{j}\right)^{\perp} h_{i+j}^{\perp}+\sum_{0 \leqslant i \leqslant n} \sum_{j \geqslant 1}(-1)^{n-i}\left(\partial e_{n-i} / \partial h_{j}\right)^{\perp} h_{i+j}^{\perp},
$$

where we use the commutator of the operator $\left(\partial / \partial h_{j}\right)^{\perp}$ with $e_{n-i}^{\perp}$. Consider the conjugate of the second group of summands in the above display. The conjugate is an operator of multiplication by the element of $\Lambda$ which is computed in the next proposition.

Proposition 3. For any $n \geqslant 1$ we have the identity in $\Lambda$

$$
\sum_{0 \leqslant i \leqslant n} \sum_{j \geqslant 1}(-1)^{n-i} h_{i+j} \partial e_{n-i} / \partial h_{j}=(-1)^{n} n e_{n} .
$$

Proof. The summation at the left hand side of (8.10) can be restricted to $0 \leqslant i<n$ without affecting the value of the sum, because $e_{0}=1$. Then by using (4.3) the sum can be rewritten as

$$
\begin{aligned}
\sum_{0 \leqslant i<n} \sum_{j \geqslant 1} h_{i+j} \sum_{|\mu|=n-i}(-1)^{\ell(\mu)} \partial h_{\mu} / \partial h_{j} \cdot \ell(\mu) ! / d_{\mu} \\
=\sum_{0 \leqslant i<n} \sum_{j \geqslant 1} \sum_{|\mu|=n-i}(-1)^{\ell(\mu)} h_{\nu} \cdot c_{\mu \nu} \ell(\mu) ! / d_{\mu}
\end{aligned}
$$

where $\mu$ ranges over all partitions of $n-i$ containing $j$ as a part. Further, here $\nu$ denotes the partition obtained from $\mu$ by replacing a part $j$ by $i+j$. According to the notation introduced after stating (8.7), here $c_{\mu \nu}$ is the multiplicity of the part $j$ in $\mu$. In particular, $\ell(\mu)=\ell(\nu)$ and

$$
c_{\mu \nu} / d_{\mu}=c_{\nu \mu} / d_{\nu}
$$

Here we extend the notation $c_{\mu \nu}$ to the case $i=0$. Then $\mu=\nu$ and the part $j$ is not determined by the notation. This should not cause us any confusion however, as it will always remain clear which multiplicity is taken. In particular, the equality (8.12) will remain valid in the case $i=0$.

By again using (4.3) the sum at the right hand side of (8.10) can be rewritten as

$$
\sum_{|\nu|=n}(-1)^{\ell(\nu)} h_{\nu} \cdot n \ell(\nu) ! / d_{\nu}
$$

where $\nu$ ranges over all partitions of $n$. To prove the identity (8.10) it remains to observe that for any fixed $\nu$ the terms at the right hand side of (8.11) containing the factor $h_{\nu}$ can be collected as follows. Choose any of the distinct non-zero parts of $\nu$ and replace it by a positive integer not exceeding the original part, hence getting some $\mu$. For any choice of the original part there are as many possible replacements as the size of the part. By multiplying the size by the ratio (8.12) and then summing over all distinct non-zero parts of $\nu$ we get $n / d_{\nu}$, exactly as in $(8.13)$.

We can now complete our proof of Theorem 3. By using Proposition 2 and then Proposition 3 as explained just before stating the latter, the sum in the second line of the display (8.8) equals

$$
\begin{aligned}
\sum_{0 \leqslant i \leqslant n} \sum_{j \geqslant 1} & \sum_{\ell(\lambda)=k}(-1)^{n-i+k+1} d_{\lambda} e_{n-i}^{\perp}\left(\partial / \partial h_{j}\right)^{\perp} h_{i+j}^{\perp}\left(m_{\lambda}\right) \otimes m_{\lambda} \\
& +\sum_{\ell(\lambda)=k}(-1)^{n+k} n e_{n}^{\perp}\left(m_{\lambda}\right) \otimes m_{\lambda} .
\end{aligned}
$$


In the first line of the above display the summation can be restricted to partitions $\lambda$ containing $i+j$ as a part, without affecting the value of the sum; see (4.5). For these partitions $\lambda$ we have

$$
d_{\lambda}\left(\partial / \partial h_{j}\right)^{\perp} h_{i+j}^{\perp}\left(m_{\lambda}\right)=d_{\lambda} c_{\mu \lambda} m_{\mu}=d_{\mu} c_{\lambda \mu} m_{\mu}
$$

where $\mu$ denotes the partition obtained from $\lambda$ by replacing a part $i+j$ with $j$; see the remark we made immediately after displaying (8.12). Therefore the summands in (8.14) with $1 \leqslant i \leqslant n$ cancel with the sum in the first line of (8.8). Since the sum of the multiplicities of non-zero parts of $\lambda$ is $\ell(\lambda)$, the summands in (8.14) with $i=0$ add up to

$$
\sum_{\ell(\lambda)=k}(-1)^{n+k+1} d_{\lambda} k e_{n}^{\perp}\left(m_{\lambda}\right) \otimes m_{\lambda} .
$$

The latter sum and the sum in the second line of (8.14) cancel with the right hand side of (8.8). All these cancellations establish the relation (8.8). Our proof of Theorem 3 is now completed.

\section{Acknowledgements}

We are very grateful to S.M. Khoroshkin and J. Shiraishi for friendly discussions. We cannot praise enough the wonderful Maple packages by J. Stembridge [17] that facilitated our work with symmetric functions. The first and the second named of us have been supported by the EPSRC grants EP/I014071 and EP/H000054 respectively.

\section{References}

[1] Ablowitz M.J., Fokas A.S., Satsuma J., Segur H., On the periodic intermediate long wave equation, J. Phys. A: Math. Gen. 15 (1982), 781-786.

[2] Awata H., Matsuo Y., Odake S., Shiraishi J., Collective field theory, Calogero-Sutherland model and generalized matrix models, Phys. Lett. B 347 (1995), 49-55, hep-th/9411053.

[3] Benjamin T.B., Internal waves of permanent form in fluids of great depth, J. Fluid Mech. 29 (1967), 559-592.

[4] Cai W., Jing N., Applications of a Laplace-Beltrami operator for Jack polynomials, European J. Combin. 33 (2012), 556-571, arXiv:1101.5544.

[5] Kaup D.J., Lakoba T.I., Matsuno Y., Complete integrability of the Benjamin-Ono equation by means of action-angle variables, Phys. Lett. A 238 (1998), 123-133.

[6] Kaup D.J., Matsuno Y., The inverse scattering transform for the Benjamin-Ono equation, Stud. Appl. Math. 101 (1998), 73-98.

[7] Krichever I., Vaninsky K.L., The periodic and open Toda lattice, in Mirror Symmetry, IV (Montreal, QC, 2000), AMS/IP Stud. Adv. Math., Vol. 33, Amer. Math. Soc., Providence, RI, 2002, 139-158, hep-th/0010184.

[8] Macdonald I.G., Symmetric functions and Hall polynomials, 2nd ed., Oxford Mathematical Monographs, The Clarendon Press, Oxford University Press, New York, 1995.

[9] Nazarov M.L., Sklyanin E.K., Sekiguchi-Debiard operators at infinity, Comm. Math. Phys. 324 (2013), 831-849, arXiv:1212.2781.

[10] Nazarov M.L., Sklyanin E.K., Macdonald operators at infinity, J. Algebr. Comb. (2013), arXiv:1212.2960.

[11] Okounkov A., Pandharipande R., Quantum cohomology of the Hilbert scheme of points in the plane, Invent. Math. 179 (2010), 523-557, math.AG/0411210.

[12] Ono H., Algebraic solitary waves in stratified fluids, J. Phys. Soc. Japan 39 (1975), 1082-1091.

[13] Polychronakos A.P., Waves and solitons in the continuum limit of the Calogero-Sutherland model, Phys. Rev. Lett. 74 (1995), 5153-5157, hep-th/9411054. 
[14] Schiffmann O., Vasserot E., Cherednik algebras, $W$-algebras and the equivariant cohomology of the moduli space of instantons on $\mathbb{A}^{2}$, Publ. Math. IHÉS 118 (2013), 213-342, arXiv:1202.2756.

[15] Shiraishi J., A family of integral transformations and basic hypergeometric series, Comm. Math. Phys. 263 (2006), 439-460.

[16] Sklyanin E.K., Bispectrality for the quantum open Toda chain, J. Phys. A: Math. Theor. 46 (2013), 382001, 9 pages, arXiv:1306.0454.

[17] Stembridge J., Maple packages for symmetric functions, posets, root systems, and finite Coxeter groups, available at http://www.math.lsa.umich.edu/ jrs/maple.html\#SF.

[18] Takasaki K., Integrable systems whose spectral curves are the graph of a function, in Superintegrability in Classical and Quantum Systems, CRM Proc. Lecture Notes, Vol. 37, Amer. Math. Soc., Providence, RI, 2004, 211-222, nlin.SI/0211021.

[19] Ujino H., Wadati M., Hikami K., The quantum Calogero-Moser model: algebraic structures, J. Phys. Soc. Japan 62 (1993), 3035-3043.

[20] Vaninsky K.L., On explicit parametrisation of spectral curves for Moser-Calogero particles and its applications, Int. Math. Res. Not. 1999 (1999), 509-529, solv-int/9808018. 